\title{
Response to ${ }^{225}$ Ac-PSMA-I\&T after failure of long-term ${ }^{177}$ Lu-PSMA RLT in MCRPC
}

\author{
Harun Ilhan ${ }^{1}$ (D) Astrid Gosewisch ${ }^{1} \cdot$ Guido Böning ${ }^{1}$ • Friederike Völter ${ }^{1} \cdot$ Mathias Zacherl ${ }^{1}$. Marcus Unterrainer ${ }^{2}$. \\ Peter Bartenstein ${ }^{1}$. Andrei Todica ${ }^{1}$ • Franz Josef Gildehaus ${ }^{1}$
}

Received: 13 June 2020 / Accepted: 31 August 2020 / Published online: 22 September 2020

(C) The Author(s) 2020

Radioligand therapy (RLT) using ${ }^{177}$ Lu-PSMA ligands is highly effective in metastatic castration-resistant prostate cancer (mCRPC); however, failure of ${ }^{177}$ Lu-PSMA RLT remains challenging as RLT already represents last-line treatment.

The $\alpha$-emitter ${ }^{225} \mathrm{Ac}$ provides higher biological effectiveness compared with ${ }^{177} \mathrm{Lu}[1]$. Several centers reported remarkable response after PSMA-targeted alpha therapy (TAT) using ${ }^{225} \mathrm{Ac}$-PSMA-617 after failure of ${ }^{177} \mathrm{Lu}-$ PSMA RLT $[2,3]$. Here we present encouraging response to TAT in a patient with advanced mCRPC showing progression after long-term ${ }^{177}$ Lu-PSMA RLT (10 cycles). PSA values are provided under the date of each PSMAPET MIP image (A-B using ${ }^{68}$ Ga-PSMA-11 and E-H using ${ }^{18}$ F-PSMA-1007). The patient was referred for RLT after radical prostatectomy and radiotherapy in 2005, and anti-hormonal therapy started in 2013 due to biochemical progression. Further progression was observed in February 2017 (A) after 2nd-line anti-hormonal therapy from 2015 to $2016,{ }^{223}$ Ra-Dichloride in 2016, and docetaxel chemotherapy from 2016 to 2017 . Two cycles

This article is part of the Topical Collection on Image of the month.

Harun Ilhan

harun.ilhan@med.uni-muenchen.de

1 Department of Nuclear Medicine, University Hospital, LMU Munich, Munich, Germany

2 Department of Radiology, University Hospital, LMU Munich, Munich, Germany of ${ }^{177}$ Lu-PSMA-617 were highly effective (B). PSA was still decreasing after two additional ${ }^{177}$ Lu-PSMA-617 cycles despite increasing PSMA-ligand uptake in PSMAPET (C). Maintenance therapy using ${ }^{177}$ Lu-PSMA-617 was continued until January 2019 with further response (D and E); however, disease progression occurred after watchful waiting and two cycles of ${ }^{177} \mathrm{Lu}$-PSMA-I\&T (F and G). The patient then received two cycles of ${ }^{225} \mathrm{Ac}$ PSMA-I\&T and showed encouraging response $(H)$. The main TAT-related side effect was grade 2 xerostomia (grade 2), which was already preexisting after 10 cycles of RLT. No TAT-related grade 3/4 hematological side effects were noted. Further cycles are planned but were suspended due to the COVID-19 crisis upon patient's request.

Different approaches including tandem therapy with ${ }^{177} \mathrm{Lu}$ or de-escalating doses during consolidation have been proposed for TAT as a trade-off between therapeutic efficacy and tolerable side effects $[2,4]$, and further studies investigating ${ }^{225}$ Ac-PSMA remain highly important for prostate cancer theranostics. 
a

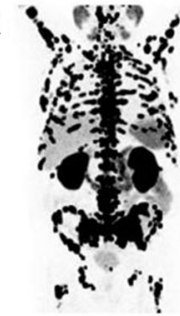

$02 / 2017$

$519 \mathrm{ng} / \mathrm{ml}$
$2 x^{177}$ LU-PSMA

RLT

(6 GBq per cycle)

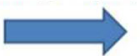

b

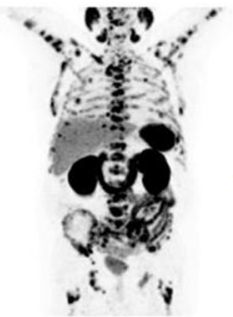

08/2017

$98.2 \mathrm{ng} / \mathrm{ml}$

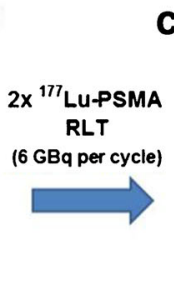

h
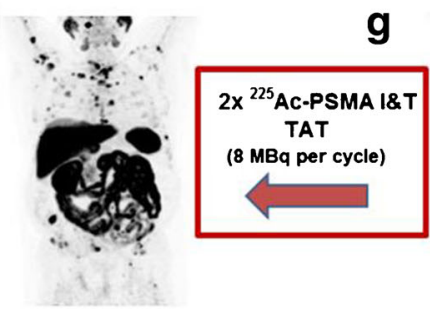

$01 / 2020$

$9.0 \mathrm{ng} / \mathrm{ml}$

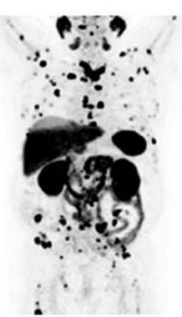

08/2019

$19.4 \mathrm{ng} / \mathrm{ml}$

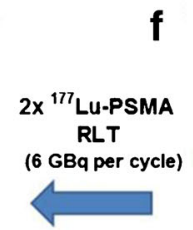

f

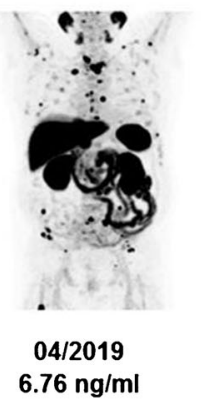

$2 x^{177}$ LU-PSMA

RLT

(6 GBq per cycle)

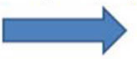

1.

A. *

$02 / 2018$

$46.8 \mathrm{ng} / \mathrm{ml}$

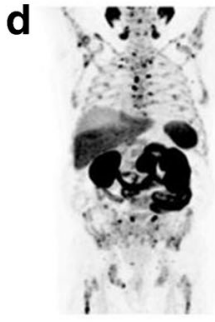

$08 / 2018$

$4.83 \mathrm{ng} / \mathrm{ml}$

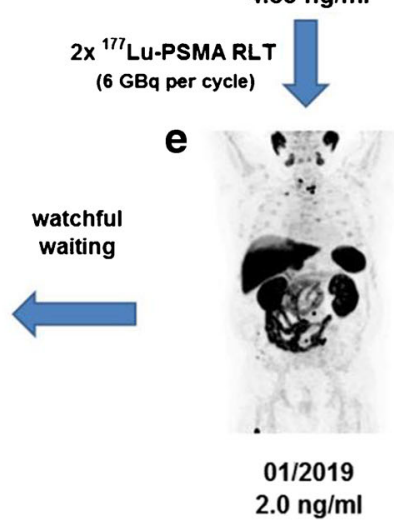

Funding Open Access funding enabled and organized by Projekt DEAL.

\section{Compliance with ethical standards}

All procedures performed in this study involving human participants were in accordance with ethical standards of the institutional and/or national research committee and with the 1964 Helsinki Declaration and its later amendments or comparable ethical standards. This retrospective evaluation was approved by the local ethic committee (20-178). Written informed consent was obtained prior to the exam.

Conflict of interest The authors declare that they have no conflict of interest.

Open Access This article is licensed under a Creative Commons Attribution 4.0 International License, which permits use, sharing, adaptation, distribution and reproduction in any medium or format, as long as you give appropriate credit to the original author(s) and the source, provide a link to the Creative Commons licence, and indicate if changes were made. The images or other third party material in this article are included in the article's Creative Commons licence, unless indicated otherwise in a credit line to the material. If material is not included in the article's Creative Commons licence and your intended use is not permitted by statutory regulation or exceeds the permitted use, you will need to obtain permission directly from the copyright holder. To view a copy of this licence, visit http://creativecommons.org/licenses/by/4.0/.

\section{References}

1. Czerwinska M, Bilewicz A, Kruszewski M, Wegierek-Ciuk A, Lankoff A. Targeted radionuclide therapy of prostate cancer-from basic research to clinical perspectives. Molecules. 2020;25. https:// doi.org/10.3390/molecules25071743.

2. Khreish F, Ebert N, Ries M, Maus S, Rosar F, Bohnenberger H, et al. (225)Ac-PSMA-617/(177)Lu-PSMA-617 tandem therapy of metastatic castration-resistant prostate cancer: pilot experience. Eur J Nucl Med Mol Imaging. 2020;47:721-8. https://doi.org/10.1007/ s00259-019-04612-0.

3. Sathekge M, Bruchertseifer F, Vorster M, Lawal IO, Knoesen O, Mahapane J, et al. Predictors of overall and disease-free survival in metastatic castration-resistant prostate cancer patients receiving (225)Ac-PSMA-617 radioligand therapy. J Nucl Med. 2020;61:629. https://doi.org/10.2967/jnumed.119.229229.

4. Sathekge M, Bruchertseifer F, Knoesen O, Reyneke F, Lawal I, Lengana T, et al. (225)Ac-PSMA-617 in chemotherapy-naive patients with advanced prostate cancer: a pilot study. Eur J Nucl Med Mol Imaging. 2019;46:129-38. https://doi.org/10.1007/s00259-0184167-0.

Publisher's note Springer Nature remains neutral with regard to jurisdictional claims in published maps and institutional affiliations. 